\title{
Morphological and spectrophotometric investigation of the layering of comet $67 \mathrm{P} /$ Churyumov-Gerasimenko
}

Gloria Tognon (1, glogeo3@libero.it), Sabrina Ferrari (1), Luca Penasa (1), Fiorangela La Forgia (2), Matteo Massironi (1, 3), Giampiero Naletto $(1,4,5)$, Monica Lazzarin (2) and the OSIRIS team

The cometary nucleus of comet $67 \mathrm{P}$ is characterized by the presence of ordered sets of morphological terraces describing a staircase morphology that allowed to infer the presence of an inner stratification (Thomas et al., 2015).

Using OSIRIS images Massironi et al. (2015) derived the orientation of the terraces and cuesta-like morphologies, by meaning of best fitting planes (upper panel) reconstructed from the stereo-photoclinometric shape model (Jorda et al., 2016) of 67P, and realized geological cross-sections of the comet (bottom panel). In addition, the calculation of the angular deviations, namely the differences between the perpendicular to the fitting planes and the local gravity vector fields unequivocally confirm the presence of an onion-like stratification enveloping independently both lobes.

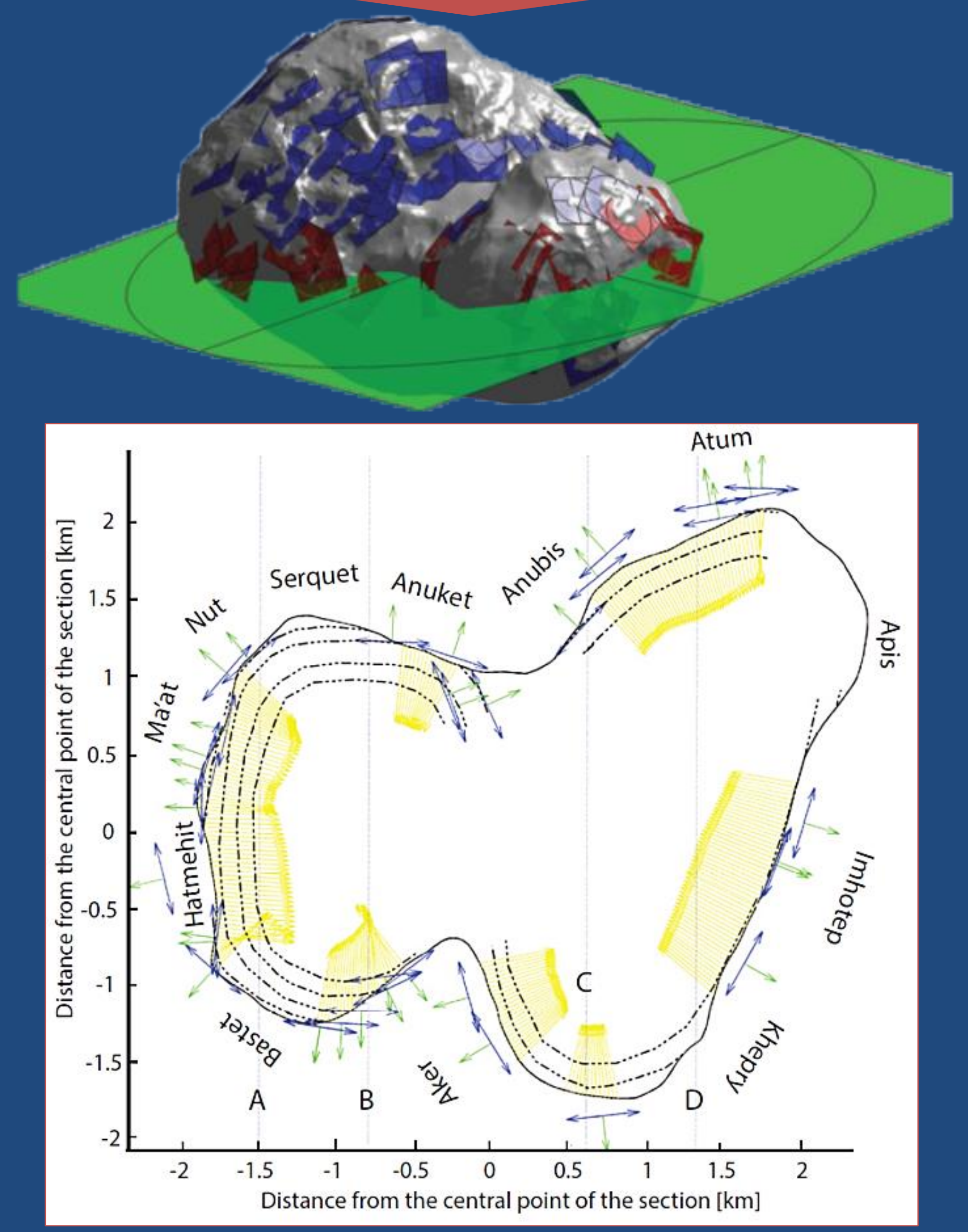

In order to distinguish different pack of layers, Ferrari et al. (2018) performed a spectrophotometric analysis of two sets of OSIRISNAC images framing physiographic regions located on the big lobe of $67 \mathrm{P}$ (left panel). The images were used to build up multispectral images photometrically corrected (La Forgia et al., 2015) to which was then applied a two-classes supervised classification on consolidated and coarse materials (central panel) on the basis of the structural elevation defined by the EM. Consolidated materials outcropping at different elevation are characterized by different brightness, in particular with the outermost layers darker than the innermost ones. Plotting the percentage of pixels pertaining to each class it is clear that increasing the structural elevation a class proportionally increases with respect to the other (right panel). The spectrophotometric variegation observed could reflect different textural properties and/or compositional differences. A similar study has been performed on the minor lobe of 67P by Tognon et al. (in preparation) obtaining results comparable with the ones concerning the major lobe.
Furthermore, by measuring the orientation of a large number of planar terraces and mesas scattered on both lobes (upper panel) by meaning of a stereo-photogrammetic shape model (Preusker et al., 2015; 2017), Penasa et al. (2017) retrieved the local orientation of the discontinuity surfaces defining the layers and reconstructed a three-dimensional geometrical model (Ellipsoidal Model, EM) based on a number of concentric ellipsoidal shells reproducing the continuation of the terraces inside the nucleus. The EM is able to accurately predict the intersection of layers with topography and to define the position (i.e. structural elevation) of any point on the cometary surface as a distance from the structural centre of the reference lobe (i.e. reference-ellipsoid semi-major axis, RES, bottom panel).
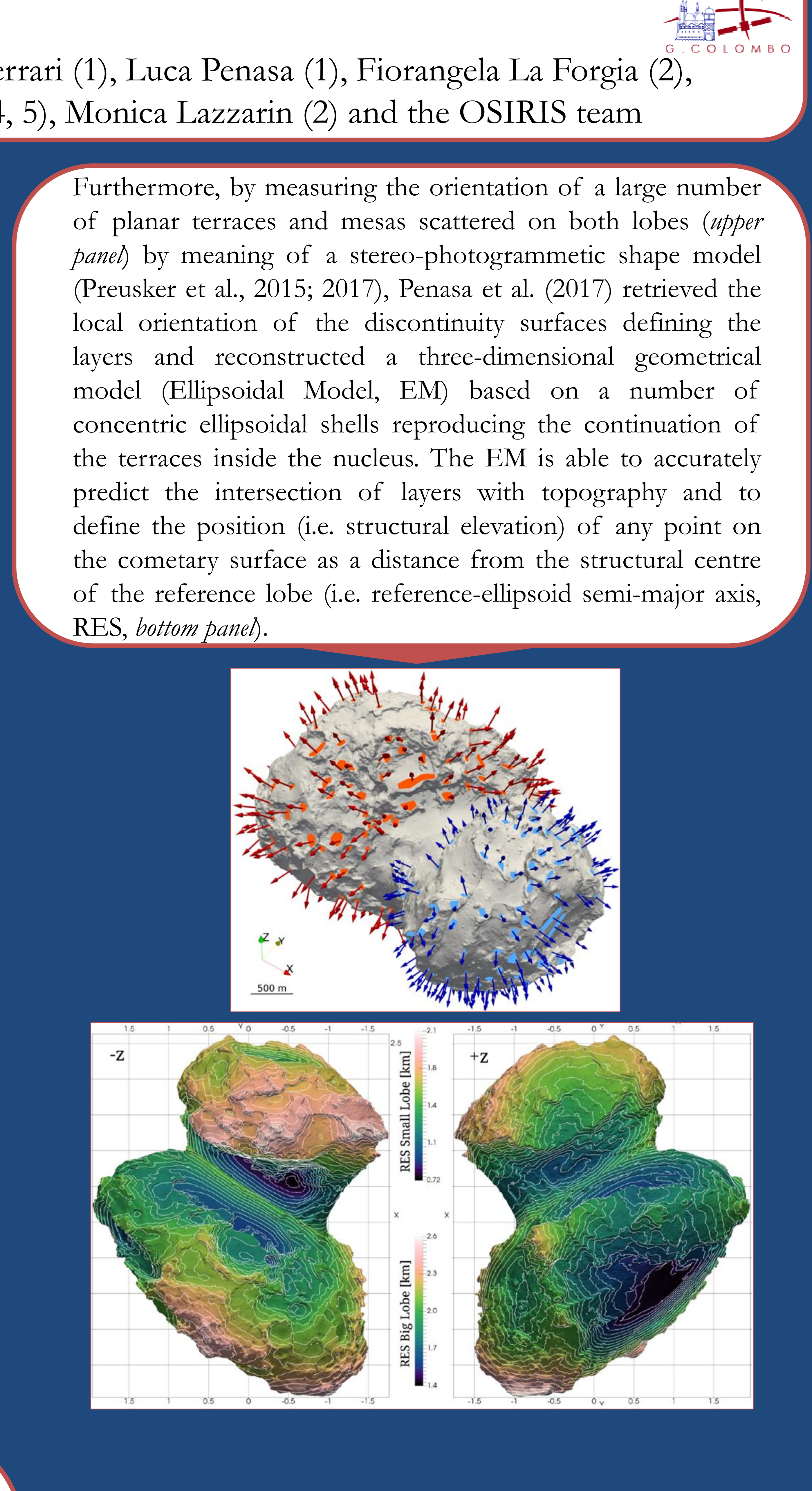

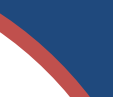
\section{.}

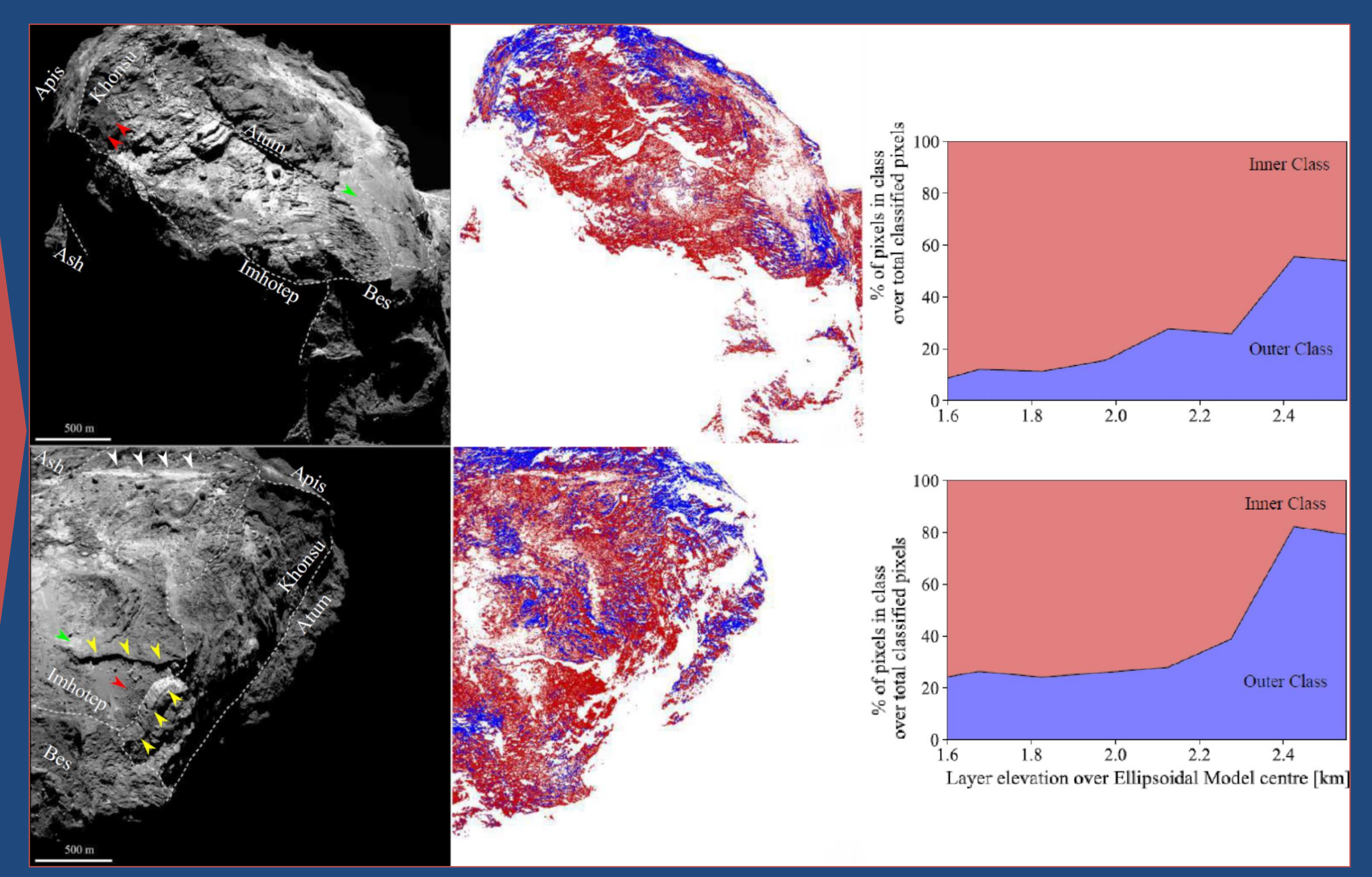

